\title{
ANALISI DELLE CARATTERISTICHE MORFOSINTATTICHE DEI DIPLOMI E CERTIFICATI DI STUDIO ITALIANI E DELLA LORO
} TRADUZIONE IN MONTENEGRINO

Deja Piletić, Università del Montenegro, dejap@ucg.ac.me

$10.31902 / \mathrm{fll} .30 .2020 .12$

UDK 371.262-057.874(450:497.16)

Il lavoro si propone di presentare i risultati della ricerca che si occupa delle caratteristiche morfosintattiche della lingua dei diplomi di laurea e certificati di studio italiani e della loro traduzione. La ricerca è stata svolta su un corpus composto da sessanta documenti originali italiani con le loro traduzioni giurate eseguite in lingua montenegrina. Oltre ai tratti morfosintattici tipici del linguaggio giuridico-amministrativo e riscontrabili nella stesura dei documenti in questione, la ricerca dovrebbe svelarci anche le caratteristiche della loro traduzione in montenegrino, nonché le corrispondenze e possibili incongruenze tra le due lingue in relazione allo stesso registro linguistico. Pertanto, riteniamo che $\mathrm{i}$ risultati presentati in questo lavoro saranno applicabili sia nella didattica della traduzione specializzata in generale, che nella prassi della traduzione giuridica in Montenegro.

Parole chiave: linguaggio giuridico-amministrativo, traduzione giurata, italiano, montenegrino, diplomi, certificati di studio

\section{Introduzione}

Il lavoro si occupa di una tipologia di testi settoriali italiani e della loro traduzione in lingua montenegrina. Si tratta di diplomi e certificati di studio, ossia di documenti ufficiali che, rilasciati dall'autorità scolastica a seguito di appositi procedimenti valutativi prescritti dalla legge, attestano il compimento di un determinato ordine di studi o il conseguimento di un'abilitazione professionale ${ }^{1}$. In quanto atti che determinano una certezza legale circa il possesso di un dato titolo di studio nel contesto nazionale, essi appartengono alla categoria di documenti che, al fine di dimostrare il loro valore legale per poter

\footnotetext{
${ }^{1}$ Cfr.http://www.treccani.it/vocabolario/diploma/; http://www.cimea.it/files/fileusers/1075_2002
} 
essere eventualmente riconosciuti all'estero, necessitano della traduzione giurata o asseverazione.

La ricerca i cui risultati vengono presentati in questa sede parte dalla considerazione riguardo ai testi specialistici, che Scarpa (104) formula in questa maniera:

"A ogni tipo di discorso specialistico corrispondono norme e convenzioni redazionali ben precise, ossia consuetudini testuali standardizzate che sono molto più stringenti che nella lingua comune, perché sono funzionali a esprimere un dato contenuto nel modo più efficiente possibile determinando per ciascuna lingua e per ciascun genere testuale l'appropriatezza linguistica in una data situazione d'uso a livello testuale, sintattico e terminologico."

$\mathrm{Ne}$ consegue che per la traduzione dei documenti al centro della nostra ricerca è indispensabile conoscere consuetudini redazionali sia del testo di partenza che di quello di arrivo.

II presente contributo, che si concentra sui tratti morfosintattici caratteristici dei diplomi e certificati di studio italiani e sulle loro traduzioni giurate in lingua montenegrina, si propone di presentare $i$ risultati della ricerca svolta su un corpus composto da sessanta documenti originali ( 25 diplomi di laurea e 35 certificati di titolo di studio) che nell'arco degli anni a partire dal 2016 al 2018 sono stati presentati al Ministero dell'Istruzione montenegrino insieme alla loro traduzione giurata come parte integrante della domanda di riconoscimento dei titoli di studio italiani in Montenegro. Tutte le traduzioni sono state eseguite da parte di traduttori/traduttrici in possesso della licenza per la traduzione giurata dall'italiano in montenegrino e viceversa per garantire che il contenuto sia conforme a quello del documento originale ${ }^{2}$.

Nella prima fase della ricerca ci siamo soffermati sull'analisi qualitativa del corpus italiano con lo scopo di individuare le caratteristiche morfosintattiche del linguaggio giuridico-amministrativo ricorrenti nella stesura di questo tipo di atti pubblici, nonché eventuali caratteristiche specifiche, non peculiari del linguaggio giuridicoamministrativo in generale. La seconda parte della ricerca è stata

\footnotetext{
${ }^{2}$ Per ottenere la licenza di traduttore/traduttrice giurato/a dal Ministero della Giustizia del Montenegro, oltre all'attestata esperienza nelle traduzioni legate al campo del diritto e oltre al superamento dell'esame inteso a dimostrare la conoscenza del sistema giuridico montenegrino, occorre superare anche degli esami che accertino la conoscenza della terminologia giuridica sia in lingua straniera che in lingua madre, nonché la competenza della traduzione e interpretazione dei testi giuridici.
} 
dedicata all'analisi qualitativa della traduzione delle strutture morfosintattiche evidenziate.

Anche se si tratta delle traduzioni professionali e nonostante sia un dato di fatto consolidato negli studi sugli "universali della traduzione" che il testo tradotto tende a orientarsi verso scelte linguistiche il più possibile vicine alla norma e alla convenzionalità della lingua di arrivo (Garzone 220), considerata la "legge di interferenza" nella traduzione, postulata da Toury (2012), ci si aspettava che i risultati dell'analisi sul corpus, rivelassero anche traduzioni meno adeguate, a conferma di quanto sostiene Garzone (218-19) ragionando sul rischio che "anche il traduttore abile, pur avendo compreso adeguatamente un testo e avendone reso correttamente il contenuto, stenda una traduzione inadeguata dal punto di vista delle scelte così dette 'di registro'"

\section{Caratteristiche morfosintattiche dei diplomi e certificati di studio italiani}

Dal momento che i titoli di studio, regolati a norma di legge, producono un effetto giuridico nella nazione in cui sono stati acquisiti e che $\mathrm{i}$ certificati e $\mathrm{i}$ diplomi che ne accertano il possesso vengono rilasciati "a tutti gli effetti di legge", questi atti potrebbero essere considerati una sottocategoria di testi giuridici.

Nella definizione di testi giuridici che propone, Garzone (196) osserva che:

"[I]n buona sostanza testi etichettati come 'giuridici' sono per lo più riferibili a generi e sottogeneri affatto diversi, ciascuno con caratteristiche proprie nel lessico, nella selezione delle strutture morfosintattiche, negli aspetti semantico-pragmatici, nella testualizzazione e nell'articolazione del discorso, con una stratificazione di gradi diversi di specificità e specializzazione, e con funzioni affatto differenti, da quella puramente informativa a quella normativa [...]."

In quanto alle categorie di testi giuridici, Mortara Garavelli (2634) propone un modello di classificazione relativo al tipo di attività che li produce - creazione delle fonti del diritto (o normazione), interpretazione e applicazione. Secondo il suo modello, quindi, i testi giuridici si possono distinguere in testi normativi, applicativi ed interpretativi. La stessa autrice tiene a sottolineare che questa divisione non deve essere considerata in modo rigido, perché le diverse attività che producono questi tipi di testo possono intersecarsi e sovrapporsi e chiarisce che i raggruppamenti interni alle tre classi non sono da considerarsi a numero chiuso. Comunque, spiega Garzone (196), nonostante le loro diverse funzioni e il diverso grado di "tecnicità", 
anche se organizzati secondo la logica del genere testuale di appartenenza, hanno in comune l'argomento - qualche aspetto del diritto. Quindi, sono necessariamente caratterizzati dall'uso di alcuni elementi tipici della lingua del diritto, sicuramente il lessico e, in misura diversa, qualche elemento strutturale.

Cortelazzo (2010) nota che, generalmente parlando, "i testi giuridici sono caratterizzati da impersonalità, concisione e ricercata distanziazione dalla lingua comune" e che "[a]l raggiungimento di questi obiettivi concorrono soprattutto le caratteristiche morfosintattiche". Queste considerazioni dovrebbero valere anche per i diplomi e certificati di studio che, secondo il succitato modello di classificazione, potrebbero essere accostati ai testi applicativi dello stile giuridico-amministrativo ${ }^{3}$.

Infatti, i risultati dell'analisi svolta sul nostro corpus della ricerca, hanno confermato la presenza di tutte e tre le caratteristiche sopra menzionate. L'impersonalità del testo si raggiunge con il prevalente uso della forma passiva e delle forme impersonali del verbo, mentre per la realizzazione della condensazione sintattica, oltre alla tendenza alla nominalizzazione (la preferenza del nome al verbo come portatore del significato), abbiamo individuato anche l'uso dello stile detto commatico e dello stile telegrafico. Quest'ultimo, individuabile soprattutto nell'omissione degli articoli e delle preposizioni, è presente sia nei certificati che nei diplomi, laddove quello commatico è stato notato soltanto nella stesura dei certificati. Questo stile, come spiega Cortelazzo (2010), sebbene sia "tipico dei testi normativi (nelle leggi il comma è costituito, tranne poche eccezioni, da una sola frase)", si estende anche a tutti gli altri tipi di testo giuridico e "prevede la corrispondenza tra capoverso e frase, intesa come unità che va da punto fermo a punto fermo". Per via del contenuto semantico, la struttura delle frasi-capoversi dei certificati di studio è molto più semplice e ridotta rispetto alla struttura delle frasi-capoversi dei testi normativi ed è spesso complementare con lo stile telegrafico. Inoltre, i

\footnotetext{
${ }^{3}$ Testi applicativi comprendono gli atti processuali (sentenze, ordinanze e decreti del giudice; requisitorie dei pubblici ministeri, orali o scritte a seconda di quanto prevedono i codici, informazioni di garanzia, impugnazioni; comparse, istanze, memorie, atti di citazione prodotti dagli avvocati, oltre alle arringhe, tipicamente orali), gli atti amministrativi (decreti, ordinanze, avvisi, verbali, ordini di servizio, proposte, pareri, visti, certificati, iscrizioni in pubblici registri), gli atti giuridici privati (contratti, testamenti, procure, copie autentiche, preliminari di acquisto, appalti, lodi).
} 
capoversi sono disposti sempre nello stesso ordine, seguendo la struttura formale prescritta.

Es. 1: Si certifica

che $X Y$, nata/o il $x x x$ a $x x x$ superò presso questa Università l'esame di Laurea in $x x x$ (classe $x x x$ - Classe delle lauree in xxx - D.M. xxx) nel giorno xxx con punti xxx su 110.

La durata normale del corso di studi per il conseguimento della suddetta Laurea di Primo Livello è di TRE anni accademici.

Per il conseguimento della predetta Laurea di Primo Livello, superò i seguenti esami di profitto, riportando le votazioni a fianco di ciascuno segnate.

Per quanto riguarda i diplomi di laurea, la struttura e la disposizione del testo è diversa. Le informazioni cruciali sono contenute in un solo periodo complesso (disposto sull'intera facciata del foglio) che consiste di almeno due subordinate implicite incidentali e inizia con la formula obbligatoria "In nome della Legge".

Es. 2:

$$
\begin{gathered}
\text { In nome della Legge } \\
\text { Noi Prof. Ing. } X Y \\
\text { Rettore dell'Università } Y \\
\text { visti gli attestati degli studi compiuti }
\end{gathered}
$$

visto il risultato della prova finale superata il giorno $x x x$ conferiamo a

$$
\mathrm{XY}
$$$$
\text { nata/o a xxx (paese) il giorno xxx }
$$

la Laurea Magistrale in

$$
\mathrm{Xxx}
$$

Nonostante le strutture formali diverse, come si può evincere dagli esempi sopra riportati, in entrambi i testi la condensazione sintattica è stata ottenuta (anche) attraverso l'uso delle forme implicite e del participio passato del verbo (Es. 1: nata/o, suddetta, predetta, riportando, segnate; Es. 2: visti, compiuti, visto, superata, nata/o).

Infine, a denotare la tecnicità del testo, oltre all'ordine "non naturale" delle parole di cui parla Raso (117), contribuisce anche la 
mancanza di articoli e di reggenze ${ }^{4}$, nonché la presenza degli stereotipi lessico-grammaticali, ossia frasi fatte ricorrenti per certe tipologie di documento in determinati contesti. Si osservi nell'Es. 2 l'uso del plurale maiestatico Noi professore... conferiamo..., che è tipico per la stesura del testo dei diplomi di laurea e denota fortemente lo scostamento dalla lingua comune.

L'analisi del corpus ha dimostrato anche un uso molto frequente degli aggettivi: seguente, presente, relativo (a volte ripetuti a breve distanza) nonché l'uso di participio passato con il valore nominale: I'interessato/a, predetto/a, suddetto/a, adito/a, citato/aanch'essi a sottolineare il carattere tecnico-specialistico del testo.

Nell'esempio n. 3 che riportiamo sotto, si possono osservare molte delle caratteristiche morfosintattiche di cui si è finora parlato forma impersonale e forma passiva del verbo, forma implicita, participio passato con il valore nominale, stile nominale, stile telegrafico, aggettivi caratteristici del testo burocratico-amministrativo (anche ripetuti a breve distanza):

Es. 3:

Si certifica che il predetto ha sostenuto la seguente prova finale:

[la] tesi di laurea e [la] relativa discussione, recante il seguente titolo: $\mathrm{xxx}$.

[il] Relatore: $X Y$

[la] Materia: Xxx

Si certifica inoltre che per il conseguimento del suddetto titolo superò i seguenti esami e/o le seguenti attività formative utili per il corso adito, riportando le votazioni indicate a fianco di ciascuno ed espresse in centesimi.

Nella tabella che segue cercheremo di illustrare con esempi (evidenziati in corsivo, quando necessario) tutte le caratteristiche morfosintattiche che abbiamo individuato come tipiche dei testi in questione.

\footnotetext{
${ }^{4}$ Omissione di articoli e di reggenze rispondono anche alle esigenze di concisione.
} 


\begin{tabular}{|c|c|}
\hline $\begin{array}{l}\text { Caratteristiche } \\
\text { morfosintattiche }\end{array}$ & Esempi tratti dal corpus \\
\hline Forma passiva del verbo & $\begin{array}{l}\text { Veduti/Visti gli attestati degli studi compiuti } \\
\text { da...; } \\
\text { Il presente certificato è rilasciato solo per } \\
\text { l'estero; } \\
\text { Il presente certificato non può essere } \\
\text { prodotto agli organi della pubblica } \\
\text { amministrazione; Imposta di bollo assolta in } \\
\text { modo virtuale; Documento rilasciato } \\
\text { mediante sistema informatico; Dagli atti } \\
\text { depositati presso questo ufficio/segreteria } \\
\text { risulta quanto segue; CFU acquisti; si rilascia } \\
\text { per gli usi consentiti dalle vigenti leggi; XY } \\
\text { immatricolato/a nell'anno accademico...; Ai } \\
\text { sensi delle modifiche introdotte dalla Legge; } \\
\text { Visti gli atti d'ufficio,...; I dati riportati nel } \\
\text { presente certificato sono } \\
\text { dall'archivio informatizzato; II presente } \\
\text { certificato contiene informazioni desunte } \\
\text { dall'archivio...; Il presente certificato viene } \\
\text { stampato elettronicamente, pertanto non è } \\
\text { consentita alcuna correzione manuale; si } \\
\text { rilascia in carta legale; ecc. }\end{array}$ \\
\hline $\begin{array}{l}\text { Forma impersonale del } \\
\text { verbo }\end{array}$ & $\begin{array}{l}\text { si certifica che...; si attesta che...; si dichiara } \\
\text { che... }\end{array}$ \\
\hline Stile nominale & $\begin{array}{l}\text { Lo studente è in possesso del titolo } \\
\text { precedente Laurea in xxx - classe delle } \\
\text { lauree in xxx; } \\
\text { Il presente certificato è inefficace fino } \\
\text { all'integrazione dell'imposta di bollo, salvo i } \\
\text { casi previsti dalla legge; } \\
\text { La durata del corso è di xxx anni per un } \\
\text { totale di xxx crediti CFU; } \\
\text { Valido per l'estero; Uso estero; ecc. }\end{array}$ \\
\hline Stile commatico $^{5}$ & $\begin{array}{l}\text { La votazione conseguita è xxx. } \\
\text { La durata legale del corso è di } 5 \text { anni. } \\
\text { Il relativo diploma è stato consegnato in }\end{array}$ \\
\hline
\end{tabular}

${ }^{5}$ L'esempio che serve a illustrare lo stile commatico è rappresentato da tre "frasi-capoversi" di un certificato riportate secondo l'ordine con cui sono disposte nel testo originale. 


\begin{tabular}{|c|c|}
\hline & data...; ecc. \\
\hline $\begin{array}{l}\text { Stile telegrafico - la } \\
\text { mancanza di reggenza }\end{array}$ & $\begin{array}{l}\text { Uso [per/all'] estero; Data [dell'] Esame; } \\
\text { Responsabile [del/per il] Procedimento } \\
\text { Amministrativo; II Capo [della] divisione; } \\
\text { Numero [del/dal] registro; N. [del/dal] } \\
\text { Verbale; N. [del] Certificato; Timbro Digitale } \\
\text { [dell'] Università X; Segreteria [per gli] } \\
\text { Studenti; Totale [di] CFU; ecc. }\end{array}$ \\
\hline $\begin{array}{l}\text { Stile telegrafico - la } \\
\text { mancanza di articoli }\end{array}$ & $\begin{array}{l}\mathrm{Di} \text { durata triennale con [la] sede } \\
\text { amministrativa presso...; } \\
\text { Contiene [le] informazioni desunte } \\
\text { dall'archivio elettronico; } \\
\text { [Le] informazioni aggiornate alla data; ecc. }\end{array}$ \\
\hline $\begin{array}{l}\text { Ordine "non naturale" } \\
\text { delle parole }\end{array}$ & $\begin{array}{l}\text { Matricola N. (invece di numero della } \\
\text { matricola) } \\
\text { Il presente certificato è composto di pagine } \\
3 \text { (invece di: composto di } 3 \text { pagine); } \\
\text {...riportando la votazione... a fianco di } \\
\text { ciascuno indicata; (invece di: indicata a } \\
\text { fianco di ciascuno) } \\
\text { Si rilascia il presente certificato in bollo } \\
\text { (invece di: il presente certificato si rilascia); } \\
\text { ecc. }\end{array}$ \\
\hline $\begin{array}{l}\text { Locuzioni preposizionali } \\
\text { tipiche di testi applicativi } \\
\text { in generale }\end{array}$ & $\begin{array}{l}\text { Ai sensi dell'art.; ai sensi e per gli effetti } \\
\text { dell'art...; a norma dell'art., ...di cui al } \\
\text { D.M...; in nome della legge; ecc. }\end{array}$ \\
\hline $\begin{array}{l}\text { Locuzioni preposizionali } \\
\text { e avverbiali tipiche del } \\
\text { linguaggio burocratico- } \\
\text { amministrativo }\end{array}$ & $\begin{array}{l}\text { a/su richiesta dell'interessato/a; alla data } \\
\text { odierna; in data; nel/il giorno; ai fini di... }\end{array}$ \\
\hline $\begin{array}{l}\text { Frasi fatte - diplomi di } \\
\text { laurea }\end{array}$ & $\begin{array}{l}\text { In nome della Legge il Rettore } \\
\text { dell'Università } X \text { conferisce la laurea in...; } \\
\text { In nome della Legge, noi Professore } X Y \\
\text { Rettore dell'Università } X[\ldots] \text { conferiamo la } \\
\text { laurea...; } \\
\text { II presente diploma viene rilasciato a tutti } \\
\text { gli effetti di legge. }\end{array}$ \\
\hline $\begin{array}{l}\text { Frasi fatte - certificati di } \\
\text { titolo di studio }\end{array}$ & $\begin{array}{l}\text { Visti/veduti gli atti d'ufficio, si certifica a } \\
\text { richiesta dell'interessato/a che...; } \\
\text { Dall'Archivio informatico del nostro Ateneo, } \\
\text { alla data odierna, risulta quanto segue...; }\end{array}$ \\
\hline
\end{tabular}




\begin{tabular}{|l|l|}
\hline & Il presente certificato non può essere \\
prodotto agli organi della pubblica \\
amministrazione o ai privati gestori di \\
pubblici servizi, a norma dell'art. 15 della \\
legge xxx; ecc.
\end{tabular}

Tabella n. 1 - Esempi delle strutture morfosintattiche tipiche dei diplomi e certificati di studio, tratti dal corpus della ricerca

Osservando la Tabella $\mathrm{n}$. 1 notiamo che la struttura morfosintattica prevalente nel nostro corpus è la forma passiva del verbo formata per lo più con l'ausiliare essere; è presente anche l'uso del si passivante (il certificato si rilascia in carta legale...) e del verbo venire (II presente certificato viene stampato elettronicamente...). Comunque, come si deduce dagli esempi sopra riportati, la forma passiva è molto spesso ellittica dell'ausiliare (imposta di bollo assolta in modo virtuale; documento rilasciato mediante sistema informatico, ecc.), mentre il participio passato può svolgere anche la funzione di subordinata implicita relativa (dagli atti depositati presso questo ufficio...) o temporale (Visti gli atti d'ufficio...). Queste scelte sono motivate da ragioni di sinteticità di cui si è già parlato prima e ci servono a conferma che una stessa struttura morfosintattica può contribuire a più di uno dei tre principali obiettivi della tipologia di testo in questione. A tale riguardo si veda per esempio la frase [per I']Uso [all']estero con cui abbiamo illustrato lo stile telegrafico (la mancanza di reggenza), ma che nello stesso tempo corrisponde perfettamente allo stile nominale - il sostantivo uso viene utilizzato al posto del verbo usare.

3. Traduzione dei tratti morfosintattici caratteristici dei diplomi e certificati di studio italiani in montenegrino

Nell'esporre i risultati dell'analisi delle traduzioni facenti parte del corpus della ricerca, ci soffermeremo su due fattori generali che, secondo la nostra opinione, hanno principalmente influenzato la loro qualità. Si tratta di due "leggi" della traduzione postulate da Toury (1995, 2012): la "legge dell'interferenza" (the law of interference) e la "legge della maggiore standardizzazione" (the law of growing strandardization). Quest'ultima, per di più, coincide con la "normalizzazione/ standardizzazione" (normalisation/ standardization) come uno degli "universali della traduzione" di cui per prima parla Baker (1993).

Secondo Toury, l'interferenza tra due sistemi linguistici nel processo della traduzione è inevitabile. Può essere più o meno 
individuabile e si manifesta come transfer positivo o negativo dal testo di partenza:

"In its most general form, the law of interference would read: in translation, phenomena pertaining to the make-up of the source text tend to force themselves on the translators and be transferred to the target text, whether they manifest themselves in the form of negative transfer (i.e., deviations from normal, codified practices of the target system), or in the form of positive transfer (i.e., an increase in the frequency of features which do exist in the target system and can be used anyway)" (Toury 2012: 310-311).

II transfer negativo che può compromettere non solo l'adeguatezza, bensì anche il senso del testo tradotto, si individua prima di tutto sul livello lessicale ${ }^{6}$. Per quanto riguarda il livello morfosintattico, il transfer negativo si manifesta per lo più nell'utilizzo delle forme morfosintattiche non naturali per la lingua di arrivo (Es. 1, 2 della Tabella n. 2), come anche nell'uso delle forme non appropriate, perché non convenzionali per lo stesso registro della lingua in cui si traduce (Es. 3, 4 della Tabella n. 2). Comunque, non comporta conseguenze negative per il contenuto semantico della traduzione.

\begin{tabular}{|l|l|l|}
\hline Es. & $\begin{array}{l}\text { La frase originale in } \\
\text { italiano }\end{array}$ & $\begin{array}{l}\text { Transfer negativo nella traduzione in } \\
\text { montenegrino }\end{array}$ \\
\hline 1. & $\begin{array}{l}\text { La durata del corso è di } \\
\text { due anni accademici. }\end{array}$ & $\begin{array}{l}\text { Trajanje studijskog programa je od } \\
\text { dvije akademske godine. } \\
\text { (al posto di: Trajanje kursa je dvije } \\
\text { akademske godine) }\end{array}$ \\
\hline 2. & $\begin{array}{l}\text { In nome della Legge noi } \\
\text { Professore XY Rettore } \\
\text { dell'Università X [...] } \\
\text { conferiamo la laurea in } \\
\ldots\end{array}$ & $\begin{array}{l}\text { Univerziteta X dodjeljujemo diplomu... } \\
\text { (al posto di: U ime zakona, profesor XY, } \\
\text { rektor Univerziteta X dodjeljuje } \\
\text { diplomu...) }\end{array}$ \\
\hline 3. & $\begin{array}{l}\text { Si rilascia per gli usi } \\
\text { consentiti dalle vigenti } \\
\text { leggi. }\end{array}$ & $\begin{array}{l}\text { Izdaje se za potrebe odobrene važećim } \\
\text { zakonima } \\
\text { (al posto di: u zakonom dozvoljenene } \\
\text { svrhe) }\end{array}$ \\
\hline 4. & $\begin{array}{l}\text { Dall'archivio informatico } \\
\text { di questo Ateneo, alla } \\
\text { data odierna, risulta } \\
\text { quanto segue: ... }\end{array}$ & $\begin{array}{l}\text { Na osnovu podataka iz elektronske } \\
\text { arhive ovog Univerziteta, na današnji } \\
\text { dan potvrđujemo kako slijedi. } \\
\text { (invece di: Na osnovu podataka iz }\end{array}$ \\
\hline
\end{tabular}

${ }^{6}$ Per un'analisi dettagliata delle traduzioni dei diplomi e certificati di studio dall'italiano in montenegrino a livello lessicale si veda Piletić (2019). 


\begin{tabular}{|l|l|}
\hline & $\begin{array}{l}\text { elektronske arhive ovog Univerziteta, } \\
\text { zaključno sa današnjim danom, } \\
\text { potvrđuje se sljedeće) }\end{array}$ \\
\hline
\end{tabular}

Tabella n. 2 - Esempi di transfer negativo, tratti dal corpus della ricerca

II transfer positivo, invece, si ha in tutti quei casi in cui le convenzioni redazionali a livello morfosintattico del testo di partenza e di quello di arrivo corrispondono: I'uso delle forme impersonali; I'uso dello stile nominale e, in una certa misura, anche l'uso della forma passiva. II transfer positivo si ha anche nelle situazioni in cui la traduzione letterale delle strutture morfosintattiche pur non essendo innaturale per la lingua di arrivo, non è neanche la più appropriata dal punto di vista stilistico (si vedano le parole evidenziate in corsivo nell'Es. 5 della Tabella n. 3).

\begin{tabular}{|c|c|c|}
\hline Es. & La frase originale in italiano & $\begin{array}{l}\text { Transfer positivo nella traduzione } \\
\text { in montenegrino }\end{array}$ \\
\hline 1. & $\begin{array}{l}\text {...salvo in casi previsti dalla } \\
\text { legge }\end{array}$ & $\begin{array}{l}\text {...osim u slučajevima predviđenim } \\
\text { zakonom }\end{array}$ \\
\hline 2. & $\begin{array}{l}\text { Si certifica che il relativo } \\
\text { diploma è in corso di } \\
\text { compilazione. }\end{array}$ & $\begin{array}{l}\text { Potvrđuje se da je odgovarajuća } \\
\text { diploma u procesu izrade. }\end{array}$ \\
\hline 3. & $\begin{array}{l}\text { La firma è omessa ai sensi } \\
\text { dell'art.... }\end{array}$ & $\begin{array}{l}\text { Potpis je izostavljen u skladu sa } \\
\text { članom... }\end{array}$ \\
\hline 4. & $\begin{array}{l}\text { Il presente certificato non } \\
\text { può essere prodotto agli } \\
\text { organi della pubblica } \\
\text { amministrazione o ai privati } \\
\text { gestori di pubblici servizi, a } \\
\text { norma dell'art. } 15 \text { della } \\
\text { legge... }\end{array}$ & $\begin{array}{l}\text { Ovo uvjerenje ne može se } \\
\text { predavati organima javne uprave } \\
\text { ili privatnim pružaocima javnih } \\
\text { usluga, shodno čl. } 15 \text { zakona... }\end{array}$ \\
\hline 5. & $\begin{array}{l}\text { Si certifica inoltre che per il } \\
\text { conseguimento del suddetto } \\
\text { titolo superò i seguenti esami } \\
\text { e/o le seguenti attività } \\
\text { formative utili per il corso } \\
\text { adito, riportando le votazioni } \\
\text { indicate a fianco di ciascuno } \\
\text { ed espresse in centesimi. }\end{array}$ & $\begin{array}{l}\text { Uz to se potvrđuje da je za } \\
\text { sticanje gore pomenute } \\
\text { kvalifikacije položio sljedeće ispite } \\
\text { i/ili nastavne aktivnosti korisne za } \\
\text { pomenuti kurs, dobivši ocjene } \\
\text { naznačene sa strane svakog od } \\
\text { ispita i izražene u tridesetinama. }\end{array}$ \\
\hline 6. & È rilasciato solo per estero. & Izdaje se samo za inostranstvo. \\
\hline
\end{tabular}

Tabella n. 3-Esempi di transfer positivo, tratti dal corpus della ricerca 
Secondo la "legge della maggiore standardizzazione" come anche secondo l'ipotesi di "normalizzazione o standardizzazione", le traduzioni tendono a utilizzare le caratteristiche lessico-grammaticali convenzionali della lingua d'arrivo. Questa tendenza, infatti, "si riferisce agli adattamenti del testo di partenza alle caratteristiche testuali più convenzionali della lingua di arrivo che le traduzioni opererebbero in modo sistematico, in modo da ottenere una lingua non marcata a livello grammaticale e collocazionale" (Scarpa 109). Per quanto riguarda il nostro corpus, la standardizzazione, intesa come adattamento alle convenzioni morfosintattiche del registro di arrivo, è più evidente nella traduzione delle frasi fatte, che nella maggior parte dei casi vengono sostituite con rispettive frasi fatte del testo di arrivo, ed è vista come una strategia positiva, considerata la tipologia del testo che si traduce (Es. 1, 2, 3 della Tabella n. 4). Questo procedimento è in linea con le strategie proposte dalle teorie della traduzione target-oriented che sono orientante verso la lingua di arrivo, ossia verso la cultura ricevente.

\begin{tabular}{|l|l|l|}
\hline Es. & $\begin{array}{l}\text { La frase originale in } \\
\text { italiano }\end{array}$ & $\begin{array}{l}\text { La standardizzazione nel testo di } \\
\text { arrivo }\end{array}$ \\
\hline 1. & $\begin{array}{l}\text { Visti gli atti d'Ufficio, si } \\
\text { certifica a richiesta } \\
\text { dell'interessata che... }\end{array}$ & $\begin{array}{l}\text { Na osnovu uvida u dokumentaciju/ Na } \\
\text { osnovu službene evidencije, na lični } \\
\text { zahtjev imenovane/imenovanog izdaje } \\
\text { se Uvjerenje kojim se potvrđuje da } \\
\text { je... }\end{array}$ \\
\hline 2. & $\begin{array}{l}\text { Il presente diploma viene } \\
\text { rilasciato a tutti gli effetti } \\
\text { di legge. }\end{array}$ & $\begin{array}{l}\text { Ova diploma se izdaje sa svim pravima } \\
\text { koja pruža. }\end{array}$ \\
\hline 3. & $\begin{array}{l}\text { La durata del corso è di } \\
\text { cinque anni accademici, } \\
\text { per un totale di 300 } \\
\text { crediti formativi } \\
\text { universitari. }\end{array}$ & $\begin{array}{l}\text { Studije traju pet akademskih godina i } \\
\text { obuhvataju ukupno 300 ECTS kredita. }\end{array}$ \\
\hline 4. & $\begin{array}{l}\text { II presente certificato è } \\
\text { composto di pagine 3. }\end{array}$ & $\begin{array}{l}\text { Ovo uvjerenje je sastavljeno od 3 } \\
\text { stranice. }\end{array}$ \\
\hline 5. & $\begin{array}{l}\text { Firma sostituita dal } \\
\text { nominativo } \\
\text { responsabile ai sensi Art. } \\
\text { xxx }\end{array}$ & $\begin{array}{l}\text { Umjesto potpisa navedeno je ime } \\
\text { ovlašćenog lica, u skladu sa članom } \\
\text { xxx }\end{array}$ \\
\hline 6. & {$[\ldots]$ recante il titolo... } & [...] pod sljedećim nazivom... \\
\hline
\end{tabular}




\begin{tabular}{|c|c|c|}
\hline 7. & $\begin{array}{ll}\text { il Rettore, veduto } & \text { il } \\
\text { risultato dell'esame } & \text { di } \\
\text { laurea superato } & \text { in } \\
\text { questa Università } & {[\ldots]} \\
\text { conferisce... }\end{array}$ & $\begin{array}{l}\text { Rektor, nakon uvida u rezultat } \\
\text { ostvaren na diplomskom ispitu } \\
\text { položenom na ovom Univerzitetu [...] } \\
\text { dodjeljuje... }\end{array}$ \\
\hline
\end{tabular}

Tabella n. 4 - Esempi dell'"applicazione" della "legge della maggiore standardizzazione"

Si noti che la standardizzazione a livello morfosintattico può comportare anche l'omissione di sintagmi del testo di partenza nel processo di traduzione, o al contrario, l'aggiunta al testo di arrivo di sintagmi non presenti in quello di partenza per far sì che la traduzione sia più vicina alle norme redazionali del tipo di testo in questione.

La standardizzazione viene adoperata spontaneamente anche nei casi in cui le strutture morfosintattiche del testo di partenza non hanno un equivalente formale nel testo di arrivo. Si pensi alla traduzione dei sintagmi con la reggenza omessa: Uso estero - za upotrebu u inostranstvu; Data esame - datum ispita; Capo divisione Šef odsjeka; Segreteria Studenti - Studentska služba, ecc.

\section{Conclusione}

Nel contributo sono stati presentati i risultati dell'analisi delle caratteristiche morfosintattiche del corpus composto dai diplomi e certificati di studio italiani e dalle loro traduzioni giurate eseguite in montenegrino.

La prima parte del lavoro è stata rivolta all'individuazione delle strutture morfosintattiche peculiari dei documenti italiani in questione, mentre nella seconda parte si è cercato di rilevare i tratti caratteristici della loro traduzione in montenegrino.

I risultati hanno dimostrato che nella stesura dei documenti oggetto della presente ricerca la scelta di strumenti morfosintattici viene dettata dalla necessità di raggiungimento di tre obiettivi principali: l'impersonalità, la condensazione sintattica e la ricercata distanziazione dalla lingua comune. Questi, come ricorda Cortelazzo (2010), sono gli obiettivi che accomunano tutti i tipi di testo appartenenti allo stile giuridico-amministrativo.

Oltre ai pochi casi di transfer negativo rispecchiato nelle traduzioni inadeguate (sia dal punto di vista della norma linguistica che delle convenzioni redazionali riguardanti il registro), i risultati dell'analisi delle traduzioni hanno rivelato la tendenza alla "standardizzazione" e una notevole presenza di transfer positivo. Mentre la "standardizzazione" viene adoperata nelle situazioni in cui le 
strutture morfosintattiche del registro giuridico-amministrativo del testo di partenza e di quello di arrivo non coincidono, il transfer positivo si riscontra in tutti quei casi in cui tra le due lingue esiste un'equivalenza formale delle strutture tipiche del registro in questione.

I rilevati casi di transfer positivo ci portano alla conclusione che tra le due lingue c'è un grado abbastanza alto di corrispondenze morfosintattiche per quanto riguarda il linguaggio dei diplomi di laurea e certificati di studio (si pensi per esempio all'uso delle forme impersonali e passive del verbo e alla nominalizzazione), mentre la "standardizzazione" come procedimento traduttivo si inserisce perfettamente nell'approccio funzionalista della traduzione e, considerata la tipologia del testo tradotto, è vista in maniera positiva.

Infatti, parlando delle caratteristiche ricorrenti del testo giuridico in generale, Garzone (218) osserva che per il lavoro del traduttore la maggiore rilevanza ha il frequente ricorso a stereotipi sintattici e lessicali e ribadisce la necessità che un traduttore sviluppi "la competenza parallela del repertorio ricorrente nonché una sensibilità sufficiente a saper produrre nella traduzione la forma corrispondente anche di espressioni in cui non ci si sia mai imbattuti in precedenza". La stessa autrice (218) spiega inoltre che:

"[i]n questo quadro particolare rilievo rivestono le collocazioni specifiche e le unità fraseologiche peculiari di questa varietà della lingua [...], cioè stilemi presenti nelle diverse lingue, ma diversamente formulati in ciascuna, sicché nella traduzione non è possibile seguirne puntualmente la strutturazione lessico-grammaticale, ma è necessario procedere sulla base della corrispondenza funzionale di interi sintagmi o proposizioni."

Considerato quanto è stato detto finora, riteniamo che $i$ risultati della presente analisi, oltre a dare un contributo agli studi contrastivi tra l'italiano e il montenegrino, potrebbero essere utilizzati nella didattica della traduzione specializzata in generale, nonché nella prassi della traduzione giurata/giuridica in Montenegro.

\section{Bibliografia:}

Baker, M. "Corpus linguistics and translation studies - Implications and applications". Text and Technology. M. Baker, G. Francis and E. Tognini-Bonelli (eds.). Philadelphia/Amsterdam: Bejamins, 1993, pp. 223-243

Cortelazzo, M. "Linguaggio giuridico-amministrativo". Enciclopedia dell'italiano

Treccani,

2010. 
http://www.treccani.it/enciclopedia/linguaggio-giuridicoamministrativo (Enciclopedia-dell\%27/taliano)/. 19 gennaio 2020

Finocchietti, C. "Il valore legale dei titoli di studio". Doc. CIMEA 108, 2002. http://www.cimea.it/files/fileusers/1075 2002

Garzone, G. "Osservazioni sulla didattica della traduzione giuridica". Tradurre le microlingue scientifico-professionali. Riflessioni teoriche e proposte didattiche. A cura di Patrizia Mazzotta e Laura Salmon. Torino: UTET, 2007, pp. 194-238.

Mortara Garavelli, B. Le parole e la giustizia. Divagazioni grammaticali e retoriche su testi giuridici italiani. Torino: Piccola Biblioteca Einaudi, 2001.

Piletić, D. "Analiza leksičkog nivoa prevoda diploma i potvrda o stečenom obrazovanju sa italijanskog na crnogorski jezik". Lingua Montenegrina, XII, 2, 2019, pp. 31-46.

Raso, T. La scrittura burocratica. La lingua e l'organizzazione del testo. Roma: Carocci Editore, 2005.

Scarpa, F. La traduzione specializzata. Lingue speciali e mediazione linguistica. Milano: Hoepli, 2001.

Toury, G. Descriptive Translation Studies - and beyond. Amsterdam/Philadelphia: John Benjamins, 1995.

Descriptive Translation Studies - and beyond. Revised edition. Amsterdam/Philadelphia: John Benjamins, 2012.

Treccani. Vocabolario dell'italiano online. http://www.treccani.it/vocabolario/.

\section{ANALIZA MORFOSINTAKSIČKIH OSOBINA JEZIKA ITALIJANSKIH DIPLOMA I UVJERENJA O STUDIJAMA I NJIHOVIH PREVODA NA CRNOGORSKI JEZIK}

Rad se bavi analizom morfosintaksičkih karakteristika jezika italijanskih diploma i uvjerenja o studijama i njihovih prevoda na crnogorski jezik. $U$ radu se iznose rezultati kvalitativne analize koja je obuhvatila korpus sačinjen od šezdeset originalnih italijanskih dokumenata sa prevodima na crnogorski jezik ovjerenim od strane sudskih tumača. Pored morfosintaksičkih osobina jezika pravno-administrativnih tekstova za koje se očekivalo da će biti uočene na istraživačkom korpusu, rezultati analize otkrivaju i specifičnosti njihovog prevoda na crnogorski jezik, kao i (ne)podudarnosti morfosintaksičkih struktura izvornog i ciljnog teksta. Stoga, očekuje se da bi rezultati predstavljeni u radu mogli 
doprinijeti kontrastivnim studijama italijanskog i crnogorskog jezika, te pronaći svoju primjenu kako u nastavi stručnog prevođenja uopšte, tako i konkretno u praksi sudskog prevođenja u Crnoj Gori.

Ključne riječi: pravno-administrativni registar, sudsko prevođenje, italijanski, crnogorski, diplome, uvjerenja o studijama 